\title{
Investigating nearby exoplanets via interstellar radar
}

\author{
Louis K. Scheffer \\ Janelia Farm Research Campus, Howard Hughes Medical Institute, 19700 Helix Drive, Ashburn, VA, USA \\ e-mail: SchefferL@Janelia.hhmi.org
}

\begin{abstract}
Interstellar radar is a potential intermediate step between passive observation of exoplanets and interstellar exploratory missions. Compared with passive observation, it has the traditional advantages of radar astronomy. It can measure surface characteristics, determine spin rates and axes, provide extremely accurate ranges, construct maps of planets, distinguish liquid from solid surfaces, find rings and moons, and penetrate clouds. It can do this even for planets close to the parent star. Compared with interstellar travel or probes, it also offers significant advantages. The technology required to build such a radar already exists, radar can return results within a human lifetime, and a single facility can investigate thousands of planetary systems. The cost, although too high for current implementation, is within the reach of Earth's economy. Received 25 March 2013, accepted 30 July 2013, first published online 12 November 2013
\end{abstract}

Key words: exoplanets, large transmitter, moons, radar, ranges, rings, surface characteristics.

\section{Introduction}

Radar astronomy has long been used to study the planets of the Solar system (Ostro 1993; Butrica 1996), returning information difficult to obtain by optical methods. It was used to reconnoiter the planets, to find their ranges accurately, and otherwise prepare for physical visits by probes. If the type of information returned by radar studies could be obtained for exoplanets, it would mark a huge increase in human knowledge.

However, at first glance, the idea of interstellar radar seems completely impractical. With our biggest radio telescopes, largest transmitters, and most sensitive receivers, we can still barely ping the moons of Saturn, about one light-hour away. Since all other stars are light years away, and the energy returned by an object at range $\mathrm{R}$ goes as $1 / R^{4}$, clearly we are a long way from interstellar radar. The gap in capability is about 20 orders of magnitude.

However, a look at the physics shows that there are no fundamental barriers to achieving a gain of this magnitude, just issues of size and cost. Furthermore, a look at the technologies of phased-array transmitters and receivers shows the costs are high but within the capabilities of Earth. This is something the human race could do now, if it was willing to expend military-budget class sums on the project. In this sense, it is much easier than interstellar travel.

In this paper, we look at what it would take to build an interstellar radar, and what the possible advantages might be.

\section{Previous work}

Williams proposed an interstellar radar in 1985 (Williams 1985), noting that the only barriers were size and cost. His suggested implementation of the transmitter was a fleet of
$160001 \mathrm{~km}$ sized spacecraft, each weighing $50000000 \mathrm{~kg}$, all inside the orbit of Mercury. A similar array spread outside the orbit of Jupiter forms the receiver; the huge baseline is used to resolve the planets interferometrically. Compared with the current work, this approach requires space-based construction well beyond the current state of the art, and would doubtless be expensive in both construction and maintenance. Also, the calculations assumed a filled aperture transmitter when computing the beam width; this might be quite difficult with the antennas in orbit.

Rzhiga, also in 1985, similarly concluded the only barriers are size and cost, and calculated that a $70 \mathrm{~km}$ antenna with a 2-TW transmitter and $10 \mathrm{~K}$ receiver could detect reflections from Earth size planets around Alpha Centauri (Rzhiga 1985). No specific means of construction were proposed, but he suggested space-based antennas to avoid problems with the troposphere. By contrast, in the current work the tropospheric fluctuations are calibrated out, allowing operation from the Earth's surface. Rzhiga also notes that the receiver array could allow interstellar transmission of television even with a modestly sized transmitting station of $100 \mathrm{KW}$ power and a $10 \mathrm{~m}$ antenna.

\section{Implementation}

The current record holder for long-distance radar observation is from Earth to Saturn's rings and its moon Titan (Muhleman et al. 1995; Campbell et al. 2003). In this paper, we will work by scaling the properties of this facility (rather than using the raw radar equations) since this already takes a multitude of practical losses into consideration. The basic parameters for Titan imaging are a one-way light travel time of $1 \mathrm{~h} 7.5 \mathrm{~min}$, a receiving antenna size of about $73000 \mathrm{~m}^{2}$, and an equivalent isotropic radiated power (EIRP) of $20 \mathrm{TW}\left[2 \times 10^{13}\right.$ watts $\left.(\mathrm{W})\right]$, 
from a transmitter power of $1 \mathrm{MW}$ and antenna gain of $73 \mathrm{~dB}$ (Renzetti et al. 1988). Each observation was about $30 \mathrm{~min}$, defined by the limited tracking time of the Arecibo telescope its Earth-fixed dish only allows it to view targets near the zenith. Longer observations are desirable, both to increase the signal-to-noise ratio and see more of the target as it rotates, but require the target to be in view of the telescope when the signal returns. A similar problem arises in the interstellar case, as we will see below.

As a first estimate, assume we wish to increase the range of Arecibo by 100000 times. This suffices to image a Titan-sized moon at 13 light years, or an Earth size planet 20 light years away, a distance that encompasses at least 100 star systems (RECONS 2012). The required product of transmitter power increase and receiving area increase is then $10^{20}$.

What is the least expensive way to get this improvement? Two factors favour improving the transmitter more than the receiver. First, using phased-array technology, an $N$ element array has $N^{2}$ times the EIRP of a single element, but only $N$ times the collecting area; so it is easier to build big transmitters than big receivers. Second, phased-array transmitters can be built with consumer electronics, do not need cooling, and do not need the costly mechanical infrastructure of receiving antennas. The difference is significant - a facility with the receiving sensitivity of Arecibo will cost somewhat between $\$ 10^{7}$ and $\$ 10^{8}$, but it seems feasible to build an Arecibo-sized transmitter for $\$ 3 \times 10^{5}$, as shown below.

To find the minimum cost, assume a total needed improvement of $G$, a receiver cost of $\$_{\mathrm{R}}$, and a transmitter cost of $\$_{\mathrm{T}}$ each, all referenced to an Arecibo-class facility. If we use $M$ receivers, we will need $\sqrt{G / M}$ transmitters. Summing the costs, then differentiating with respect to $M$ gives the least expensive solution as $M=\left(1 / 2 \$_{\mathrm{T}} \$_{\mathrm{R}}\right)^{2 / 3} G^{1 / 3}$. Assuming $\$_{\mathrm{R}}=10^{8}$, $\$_{\mathrm{T}}=3 \times 10^{5}$ and $G=10^{20}$, we find a minimum cost at 61000 Arecibos receiving and $41 \mathrm{M}$ Arecibos transmitting. The minimum cost is quite broad, ranging from 40000 to 100000 Arecibos for receiving for less than a $10 \%$ variation in cost. Since huge receivers have a wider range of uses than giant transmitters, we bias our solution slightly to the large receiver side, and will assume a transmitter $10^{15}$ times more powerful and a receiver roughly $10^{5}$ times more sensitive than Arecibo.

The first step is to define the signal we wish to transmit. Historically, a variety of different frequencies have been used for planetary radar (Butrica 1996). For earth-bound observations, the allowable frequencies are defined by the atmospheric microwave window, which extends very roughly from 1 to $20 \mathrm{GHz}$. The scientific utility depends little on the exact wavelength; so the frequency of operation is driven more by the availability of powerful transmitters and sensitive receivers. The two largest facilities, Arecibo and Goldstone, use $\mathrm{S}$ band $(2 \mathrm{GHz})$ and $\mathrm{X}$ band $(8 \mathrm{GHz})$, respectively. From the transmitter's point of view, higher frequencies result in higher antenna gains. At a constant power density (in $\mathrm{W} \mathrm{m}^{-2}$ ), the EIRP attainable at frequency $f$ scales as $f^{2}$, favouring the use of higher frequencies. From the receiver's point of view, frequency has little effect on sensitivity - since the radar return is not resolved, the most relevant characteristics are collecting area and noise temperature. However, the receiver must contend with noise introduced by the atmosphere, which begins to rise above $10 \mathrm{GHz}$. Here, we assume $15 \mathrm{GHz}$ as the compromise chosen.

Independent of the frequency, circular polarization is almost always used for modern planetary radar, since it reveals information about the surface type and texture. For example, terrestrial planets are dominated by the same-sense reflection, but icy moons return a much larger fraction of echo power in the opposite sense (Butrica 1996, Chap. 8).

On the receiving side, the most straightforward approach is to build 100000 Arecibos, for a total collecting area of about $7300 \mathrm{~km}^{2}$. The antennas combined would require an area of about $10000 \mathrm{~km}^{2}$ or an area $100 \mathrm{~km}$ on a side. This is certainly possible in the more remote desert areas - for example, a Radio Quiet zone of the required size already exists in the interior of Australia (ACMA 2012). However, it would be much better to spread the array over a wide geographic area. This would improve resolution when used as a radio telescope, and reduce the potential effect of weather-induced loss when the signal returns.

Although this approach gives the required sensitivity, it has serious disadvantages. First, it may be hard to extend this approach to frequencies in the high end of the microwave window, which we noted is desirable for minimizing the physical size of the transmitter. Next, it cannot view the entire sky, leaving many interesting systems inaccessible. Finally, it would require the echo to return within a few hour window of the Earth's 24 h day. This depends on the distance to the target, which we cannot control, and would further reduce the number of systems that could be studied at any given time.

A better approach is probably an array of steerable dishes. The total area needed would be somewhat less than the multiple Arecibo case, since Arecibo does not use the whole aperture at any given time - some margin is needed so steering the feed does not cause the pattern to leave the dish. The active area is an oval $213 \times 237 \mathrm{~m}$ (Goldsmith 1996), for an area of about $40000 \mathrm{~m}^{2}$. The same collecting area would be obtained with an array of 6 million $30 \mathrm{~m}$ antennas, or 36 million $12 \mathrm{~m}$ dishes or other combinations ${ }^{1}$.

In any of these schemes, the signals from all the antennas must be combined in phase. This requires calibration of facilities and tracking of atmospheric and ionospheric delays. Using modern electronics and time standards, this is a routine operation for today's VLBI imaging. Combining radar returns is easier than imaging, since it is only concerned with an unresolved point source of known frequency, narrow bandwidth and known location. Therefore the signals can simply be summed (after appropriate delays) and multiple pairwise correlations are not required. Phase tracking through the unknown atmosphere is much simpler as well; the effort

\footnotetext{
${ }^{1}$ For radar, only the total collecting area and noise figure are important, so this tradeoff can be made freely. This is very different from radio astronomy, where U-V coverage, correlator design, field of view, frequency coverage, and many other issues are all complicate the issue of dish size selection.
} 
required is linear in the number of antennas and will not be a significant portion of the cost (Rogstad 2005). Finally, each observation is guaranteed to have an ideal calibration source - the host star of the planet. It is very near on the sky, well within the primary beam, and a strong source for an array of this size - the current VLA can see even quiet stars out to 1.4 parsecs (Drake 1993).

On the transmit side, one possibility might be an array of $10^{15}$ small antennas, each radiating a $10-\mathrm{mw}$ signal, circularly polarized, into a half hemisphere. If correctly summed in phase, this generates an EIRP of $2 \times 10^{28} \mathrm{~W}$, about $10^{15}$ times the strength of Arecibo, as required. Assuming a wavelength of $2 \mathrm{~cm}(15 \mathrm{GHz})$ and elements spaced $\lambda / 2$ or $1 \mathrm{~cm}$ apart, the array is about $316 \mathrm{~km}$ on a side. Such an array might be constructed from $10^{13}$ tiles, each about $10 \mathrm{~cm}$ on a side. The bottom of each tile has a metallic ground plane, and on the top are printed 100 antennas. In the centre of the tile there is a single chip that transforms $2 \mathrm{~W}$ of DC into 100 separate $10 \mathrm{mw}$ radio frequency (RF) signals with $50 \%$ efficiency. Each tile must know its location and orientation, and a vector to the target, and then be able to independently adjust the phase of each of its antennas. These tasks are well within the capabilities of modern electronics (Scheffer 2005).

As with receiving arrays, the main technical challenge is maintaining phase coherency and a number of solutions have been proposed (Davarian 2007). To keep the phasing losses small, the locations of the tiles must be known to mm accuracy for $2 \mathrm{~cm}$ wavelength operation (Ruze 1966). To avoid the need for surveying, each tile can implement a triangulation scheme similar to that of the Global Positioning System (GPS) in order to determine its own location and orientation. Existing GPS techniques already come close to the needed performance, demonstrating $4-10 \mathrm{~mm}$ accuracy across $1000 \mathrm{~km}$ (Dixon 1991), and the required $\mathrm{mm}$ accuracy over shorter baselines (Alber et al. 1997). Similar techniques, implemented near the frequency of array operation, should be able to meet the required accuracy. The main advantages over GPS would be higher signal strength (there is no need to cover the whole Earth) and higher-frequency operation (which improves the accuracy derived from phase comparison). Furthermore, using common tile antennas for receive and transmit will allow many source of uncertainty to be calibrated out.

The next requirement for the transmit array is to calibrate out the atmospheric delays. Unlike the receiver array, where phasing can be done in post-processing, this adjustment must happen in real time. Likely best for an array of this size would be a space-based calibrator, operating within a few degrees of the target. It could broadcast to the array on nearby harmonically related frequencies, such as $9 / 10 f_{0}$ and $11 / 10 f_{0}$, where $f_{0}$ is the nominal transmit frequency. This would allow each receiver to derive a common time and frequency reference, and measure and compensate for ionospheric and atmospheric delays at the frequency of interest. It can also distribute the information needed by all tiles, such as the direction of the target beam and the modulation strategy. Furthermore, assuming a space-based calibrator, portions of the array could be directed to transmit to the spacecraft instead of the target, allowing direct measurement of the transmitter phase after transiting the atmosphere.

Powering such an array is an excellent application for solar power. It would be acceptable to run such a transmitter only during daylight hours and clear weather, so there is no need for energy storage. The power can be utilized right where it is produced, so there is no need for distribution. Finally, the electronics need low-voltage DC, which is exactly what solar cells produce - there is no need for energy conversion. Therefore for this analysis we will assume that the $2 \mathrm{~W}$ of DC comes from a $20 \%$ efficient solar cell coating the tile.

The total amount of RF energy is about $10 \mathrm{TW}$, which (during the day) is roughly $1 / 10$ of the energy of the sunlight falling on the array. The outgoing beam is only $1 / 10$ the intensity of sunlight and hence would not be instantly destructive to airplanes and satellites that might cross its path, although precautions would surely be advisable. Unlike the receiver array, the transmitter should be as compact as possible; otherwise beam width, already rather small, becomes a serious limitation. Unlike the receiving array, there is no need to locate this facility in a Radio Quiet zone.

\section{Cost}

What about the cost? This section uses US\$ as of 2013, with suffixes $\mathrm{M}=$ million $\left(10^{6}\right), \mathrm{B}=$ billion $\left(10^{9}\right)$ and $\mathrm{T}=$ trillion $\left(10^{12}\right)$.

Arecibo cost $\$ 9.7 \mathrm{M}$ to build in 1960 , with upgrades of \$8M in 1974 and \$27M in 1997 (Matthews 2012). A similar but modernized telescope (FAST in China) is being constructed today, and is expected to cost about 600 million RMB or about $\$ 100 \mathrm{M}$ (Nan 2008); 100000 of these would cost about $\$ 10 \mathrm{~T}$, assuming enough suitable terrain can be found. (This style of telescope is most easily built in a pre-existing natural depression.) Alternatively, $12 \mathrm{~m}$ dishes are currently about $\$ 300000$ each (CSIRO 2008); 36 million of these would total about \$11T. A third alternative would be to replicate the SKA, which is aiming for a square kilometre of collecting area for a cost of about \$2B (Dewdney et al. 2009). About 4000 of these would cost $\$ 8 \mathrm{~T}$.

All of these prices are probably overestimates, since no-one has yet tried ordering radio telescopes in bulk quantities suitable for mass production. For manufactured items in general, each doubling of the volume typically reduces cost (for electronics and manufacturing) to somewhere between 90 and $95 \%$ of the original cost (Yelle 1979; Malerba 1992). For the volumes considered here, with normal learning curves the cost per item will be several times cheaper by the end of the production run.

On the transmitter side, as of 2012, you can purchase toys for considerably less than $\$ 10$, more than $10 \mathrm{~cm}$ on a side, with significant RF electronics content. A tile is simpler than many of these toys - just a slab of plastic, metallized on one side, with the other containing a Solar cell, a chip, and an array of printed antennas. With a bulk order of $10^{13}$ tiles, a cost of $\$ 1$ or less seems reasonable. Installation could be as simple as strewing 
the tiles across a desert landscape - using GPS style triangulation, each tile could determine its own location, orientation and a common time reference. It could then respond to broadcast commands telling what signals to transmit. Compared with the receiving side, the expected saving from mass production would be less on the transmitting side, since the most costly components, the solar cells and chips, are already in mass production.

Combining the transmitter and receiver gives a total cost of \$20T or less. If built over a 10-20 year period, this is far above any current science budget, but is comparable with spending on consumer electronics or militaries - the global electronics industry is about \$1T per year, and all defence spending combined is about $\$ 1.2 \mathrm{~T}$ per year. Alternatively, this would be a 20 -year project at $\$ 140 /$ year for each person on earth, or $\$ 1000 / y e a r$ for each person in a developed economy. So, humanity could do this project today if it felt the need.

\section{Aiming and listening}

There are two problems with interstellar radar that make accurate knowledge of positions and ranges mandatory. First, the beam has to hit the planet, and second we need to know when to expect the return.

Hitting the target with the beam is non-trivial since the beam is small, a necessary consequence of the high flux-density needed. In the case proposed here, the transmitter is about $1.6 \times 10^{7} \lambda$ on a side, so the natural beam width is about 0.013 arcseconds. (Thus for the interstellar case, as well as within our Solar system, radar is a poor search tool and much better suited to following up existing discoveries.) The good news is that the beam is large enough to illuminate any moons or rings of the planet it is targeting.

The location of the star on the sky, and its proper motion, must be known well enough to predict the location of the star when the beam gets there. Furthermore, we need to know where the planet is with respect to the star, since it can range up to hundreds of beam widths away. A 1 AU orbit at 1 parsec range may appear up to 1 arcsecond away from the star, so the orbit must be known to roughly $1 \%$ accuracy. The alternative of covering an uncertain location by multiple pointings is unpalatable, since it reduces the productivity of a very expensive facility by the number of pointings required.

In general, existing techniques such as radial velocity, astrometric or transit observations will not yield an unambiguous orbit of the required accuracy. Instead, a combination of techniques will be needed (Shao et al. 2010), almost certainly including direct imaging techniques such as those proposed for the Terrestrial Planet Finder (Levine et al. 2009; Martin et al. 2011). These are aiming at $10 \%$ accuracy on systems up to $30 \mathrm{pc}$ distant (Beichman et al. 2006), and should provide the required accuracy on the nearer systems considered here.

In addition to the position, the distance to the system must be known quite accurately, for two reasons. The first requirement is to hit the planet, which is a moving target. This requires knowledge of how long the signal will take to get there. The second reason, requiring yet more accuracy, is to know when to look for the return signal. This is potentially a more troubling problem, particularly if the receiving array has rotated out of view when the signal returns. There are at least three potential solutions to this problem. One possibility would be to build three facilities spaced around the world, as JPL does with the Deep Space Network, though this would be expensive. Alternatively, the receiver could be space based and capable of $24 \mathrm{~h}$ operation, although this would be more expensive yet. The simplest solution would be just to wait. A typical radial velocity for a nearby star is about $10 \mathrm{~km} \mathrm{~s}^{-1}$ (Reid et al. 2007). At this rate, the return time will change at about $2000 \mathrm{~s}$ year $^{-1}$. Since the worst case would require a $12 \mathrm{~h}$ change, we can get optimal reception within 20 years or so. Unfortunately there still may be a few stars with both low radial velocities and inconvenient round-trip times for which waiting is not a practical solution.

For return-time calculations, the distance to the star must be known to a few light hours. This will need to be established through parallax measurements. At 20 light years, this implies a parallax accuracy of roughly $7 \mu$ as. This accuracy can be attained by Gaia technology (Lindegren 2009), although Gaia itself is not sufficiently accurate on stars this bright since they saturate the detectors (Gardiol et al. 2005). Once an initial signal is received, uncertain ranges will no longer be a problem - the uncertainty in distance immediately drops from a few light hours to a light millisecond or less. That is an improvement of $10^{8}$ or so in one observation.

\section{Transmission}

Different radar techniques and modulations are strongly preferred depending on the rotational speed and size of a target (Harmon 2002). Sizes and rotation rates vary widely even within the Solar system, from targets such as Mercury that are compact and rotate slowly, to asteroids that are small and rotate quickly, to Saturn's rings, which are large and orbit with high velocity. Within the Solar system, different radar techniques are used for each target type.

For extrasolar planets, rotation rates, sizes and the presence of rings and moons may all be unknown until we get the echos. Rather than waiting another round trip time to try again, it probably makes sense to probe each planet with a set of ranging waveforms. These should be picked so at least one gives decent results over the range of expected planetary characteristics.

A single radar observation can reveal the target size from the range of delays. It also shows the rotational velocity, projected onto the line of sight, from the doppler shift, but it does not show the rotational axis, which is important for both theoretical studies of Solar system evolution and potential habitability. However, if features can be resolved this can be measured by repeated observation. An accurate orbit of the planet around its parent star needs several separated observations as well. Therefore rather than a single long observation, it may be better to observe a number of times over a range of hours, days, weeks, months or even years. This range 
of times (if the Solar system is typical) should allow determination of accurate orbits and axial tilts.

\section{Strategy}

The number of potential targets rises with range $R$, in theory as $4 / 3 \pi R^{3} d$ where $d$ is the local density of stars. In practice, near Earth, each additional light year in range buys you just one to two stars at the beginning, rising to 15 additional stars per year of range at 20 light years. Each additional light year of distance requires two more years of round trip time. In perhaps the simplest scheme, the transmitter starts by illuminating the planets around the nearest star, then switches to the next further star when twice the distance between them (in light time) has elapsed. This keeps both the transmitter and receiver busy all the time. For these nearby stars, several months per star will be available for analysis.

There is no reason, except economics, that this method could not be extended out further than the initial 20 light years. There is no high energy-density to cause trouble with material properties, and the only technologies needed are precision timing, accurate positioning and mass production. A transmitter $3000 \mathrm{~km}$ in size would have 100 times the number of transmitters, and 10000 times the EIRP, leading to a range $10 \times$ greater. This would be about 130 light years for moons and 200 light years for planets, encompassing at least 6000 known star systems (Nash 2011).

As the range increases, there will be more systems at similar distance, and hence less time per system. A reasonable minimum might be 1 day per system, which will happen when the range is such that each additional 1/2 light year of radius incorporates 365 new systems. This will happen somewhere about 100 light years, and past this range only a subset of targets can be examined.

\section{Advantages}

There are many advantages to radar observations. When compared to interstellar probes or missions, one big advantage is that results will come within in a human lifetime. For example, a radar observation of $\tau$ Ceti will be complete 25 years after the signal is sent. This is already less elapsed time than some deep space missions such as Voyager, which are still returning data 35 years after their launch. For comparison, a probe, even at an optimistic $10 \%$ of the speed of light, will take 125 years to get there, and another 12 years to send the signal back. Therefore any probe sent will only return data to an investigator's descendants.

Another advantage is that a radar facility can observe hundreds to thousands of nearby systems in detail, including many interesting targets such as $\tau$ Ceti (suspected to have five planets (Tuomi et al. 2012)) and Epsilon Eridani. Each probe, in contrast, will only observe one system. To be fair, however, some probe architectures, such as a large launching facility that sends out lightweight 'starwisp' style probes, offer similar benefits.

The very first echos obtained from an exoplanet will tell if the planet is rocky, icy, or has a liquid surface. This is because rock and ice reflect polarized EM waves differently (Butrica 1996), and liquids give a specular reflection from their smooth surface (Campbell et al. 2003). Reflection of polarized waves also allows various physical properties to be inferred, such as surface roughness on the scale of a wavelength or greater, or potential dielectric constants of the reflecting material (Simpson et al. 1992). This type of analysis has been used (along with orientation information) to infer the presence of ice at the poles of the Moon (Stacy et al. 1997) and Mercury (Harmon et al. 2001), and investigate properties of the ice-caps of Mars (Harmon et al. 1999).

Radar imaging generates crude images of planets by means of 'delay-doppler' mapping. Unlike conventional imaging, the available resolution depends only on the signal to noise of the echo, but not on the physical resolution of the beam or the distance to the planet. Titan has been mapped with 10 degree resolution (hundreds of pixels) (Black 2010), with the Moon (Zisk et al. 1974; Thompson 1987), Venus (Rogers et al. 1969), Mercury (Butler et al. 1993) and Mars (Harmon et al. 1999) mapped to much higher precision. Obtaining even low resolution maps for exoplanets will be difficult with other techniques. The maps obtained, however, will still be NorthSouth ambiguous, for at this range interferometric disambiguation (Butrica 1996) will not be possible.

Radar observations can perform detailed studies of planets close to their suns, difficult by other means. In particular, optical methods have trouble analysing close-in planets that do not eclipse their star, since they are very difficult to resolve against the nearby and much brighter star. This problem is sufficiently serious that even in our own Solar system, the spin properties of Mercury had to be determined by radar (Pettengill \& Dyce 1965).

Radar measurements will immediately improve ranges to other systems by many orders of magnitude. They will also improve velocity with respect to the Solar system, although by not as much since a great deal of work has already gone into precise radial velocities. Both the distance and the velocity will be very helpful when sending a probe.

Radar investigations of a planet will also show the presence of moons and/or rings. If they are observed several different times the detailed orbits can be inferred.

Radar can also determine the rotation rates for all bodies, and their axis of rotation. In the Solar system, this was used to determine the rotation rates of Mercury and Venus - Mercury because it is close to the sun and hard to observe optically, and Venus because it is shrouded by clouds. Both of these situations are likely to occur in other Solar systems.

For SETI, a potential advantage is the prospect of detecting civilizations that are not trying to communicate, and perhaps ones that have self-destructed, depending on the durability of their structures. Cities, for example, are well known to have much brighter radar echos than natural surfaces, since they contain many flat surfaces at right angles, a geometry that contributes to strong radar reflections (Long 1975). They also exhibit several other 'unnatural' reflectivity properties, since as strong reflections at incidences grazing the surface (due to vertical walls) and stronger reflections when aligned with the 
compass directions (Kayton \& Fried 1997) (for Earth cities, anyway).

Another possibility is that civilizations could leave very long lasting and easy to find radar artefacts. Radar reflectors are one of the few examples where objects of size typical of human construction may be visible across interstellar distances. The radar cross-section of a trihedral (corner reflector) scales as the fourth power of the side length $a$ :

$\sigma=\frac{12 \pi a^{4}}{\lambda^{2}}$.

A trihedral reflector $120 \mathrm{~m}$ on a side would provide a $15 \mathrm{GHz}$ radar reflection as strong as that of a $5000 \mathrm{~km}$ diameter moon. If this trihedral was cut into a planet's moon, this echo would blink on and off as the moon rotates; an effect that would surely draw attention to its likely artificial nature. An advantage of such an artefact is that no aiming or maintenance is required, and it could outlast the civilization that created it by many millions of years.

\section{Other uses}

There are many other possible uses for a system of this size. Clearly the receiver portion would make a great radio telescope. The transmitter part can be used to blast debris out of Earth orbit, or provide power to far away probes.

The radar could image any object within the Solar system, Kuiper belt or Oort cloud, although an alternative receiver would need to be used for some particular light travel times, for which the echo returns when the main receiver is out of view.

The transmitter would make a fine interstellar beacon, if Earth chooses to use it that way. Integrated over all bandwidths, it outshines the sun by a factor of 50 - the Sun has an EIRP of $4 \times 10^{26} \mathrm{~W}$. In the radio regime, it is extremely bright by radio astronomy standards, where a strong source may have a flux of 10 Jansky (Laing et al. 1983). Integrated over a $1 \mathrm{GHz}$ bandwidth, this gives $10^{-16} \mathrm{~W} \mathrm{~m}^{-2}$. By comparison, the source here would deliver roughly $1.8 \times 10^{-7} \mathrm{~W} \mathrm{~m}^{-2}$ at a distance of 10 light years, so it is roughly 2 trillion times $(93 \mathrm{~dB})$ brighter than the typical bright target for a radio telescope. A radio telescope would not need to be pointed at the transmitter to see this signal; it would not even need to be pointed anywhere in the general direction. Unless it is unusually well shielded, simply being turned on, and on the right side of the planet, should suffice. On the other hand, for any particular target of the radar investigation, the signal will be highly intermittent, and may never re-occur. Another implication for SETI is that you cannot necessarily assume that a signal is man-made just because it is strong or visible in the side-lobes of the antenna.

If after a radar investigation, Earth sends a probe or probes, the facility can serve as uplink and downlink.

\section{Conclusions}

Interstellar radar can give considerable information about nearby exoplanets that cannot be obtained through optical means. It is within the range of Earth's technology and economy even now. Although quite expensive, it is surely cheaper than an interstellar probe, and would make an excellent intermediate step.

\section{References}

ACMA (2012). Planning for the Radio Astronomy Service. URL: http:/ www.acma.gov.au/WEB/STANDARD/pc $=$ PC100628

Alber, C., Ware, R., Rocken, C. \& Solheim, F. (1997). GPS surveying with $1 \mathrm{~mm}$ precision using corrections for atmospheric slant path delay. Geophysical Research Letters 24(15), 1859-1862.

Beichman, C.A., Johnston, K.J., Bally, J., Kaltenegger, L., Rttgering, H., Serabyn, E. \& Crisp, D. (2006). Terrestrial Planet Fnder Interferometer -Planetary Detection and Characterization. URL: http://exep.jpl.nasa.gov/ TPF-I/scienceRequirements.cfm

Black, G. (2010). A global map of Titan's $13 \mathrm{~cm}$ wavelength radar reflectivity, in Bulletin of the American Astronomical Society, vol. 42, p. 1064.

Butler, B., Muhleman, D. \& Slade, M. (1993). Mercury: full-disk radar images and the detection and stability of ice at the north pole. Journal of Geophysical Research 98(E8), 15003-15015.

Butrica, A. (1996). To See the Unseen: A History of Planetary Radar Astronomy. p. 1. National Aeronautics and Space Administration, Washington, DC.

Campbell, D., Black, G., Carter, L. \& Ostro, S. (2003). Radar evidence for liquid surfaces on Titan. Science 302(5644), 431-434.

CSIRO (2008). CSIRO Lets Major Antenna Contract for ASKAP. URL: http://www.atnf.csiro.au/projects/mira/ASKAPantenna.pdf

Davarian, F. (2007). Uplink arrays for the deep space network. Proceedings of the IEEE 95(10), 1923-1930.

Dewdney, P., Hall, P., Schilizzi, R. \& Lazio, T. (2009). The square kilometre array. Proceedings of the IEEE 97(8), 1482-1496.

Dixon, T. (1991). An introduction to the Global Positioning System and some geological applications. Reviews of Geophysics 29(2), 249-276.

Drake, S. (1993). Radio emission from coronal-type stars. In Physics of Solar and Stellar Coronae: GS Vaiana Memorial Symposium, pp. 393-400. Springer, Palermo, Italy.

Gardiol, D., Busonero, D., Gai, M. \& Lattanzi, M. (2005). Gaia performance on bright stars. In $S W G 7$ Meeting, pp. 2-4. Paris.

Goldsmith, P. (1996). The second Arecibo upgrade. Potentials, IEEE 15(3), 38- 43.

Harmon, J. (2002). Planetary delay-doppler radar and the long-code method. Geoscience and Remote Sensing, IEEE Transactions on 40(9), 1904-1916.

Harmon, J., Arvidson, R., Guinness, E., Campbell, B. \& Slade, M. (1999). Mars mapping with delay-doppler radar. Journal of Geophysical Research 104(E6), 14065-14089.

Harmon, J., Perillat, P. \& Slade, M. (2001). High-resolution radar imaging of Mercury's north pole. Icarus $\mathbf{1 4 9 ( 1 ) , 1 - 1 5 .}$

Kayton, M. \& Fried, W. (1997). Avionics Navigation Systems. WileyInterscience, New York, New York, USA.

Laing, R., Riley, J. \& Longair, M. (1983). Bright radio sources at 178 mHzflux densities, optical identifications and the cosmological evolution of powerful radio galaxies. Monthly Notices of the Royal Astronomical Society 204, 151-187.

Levine, M., et al. (2009). Terrestrial Planet Finder Coronagraph (TPF-C) flight baseline concept, arXiv: 0911.3200.

Lindegren, L. (2009). Gaia: astrometric performance and current status of the project. In Proceedings IAU Symposium No 261, Relativity in Fundamental Astronomy. Cambridge University Press, Virginia Beach, Virginia, USA.

Long, M. (1975). Radar Reflectivity of Land and Sea, p. 1. DC Heath and Co., Lexington, Mass.. 390.

Malerba, F. (1992). Learning by firms and incremental technical change. Economic Journal 102, 845-859.

Martin, S., Ksendzov, A., Lay, O., Peters, R.D. \& Scharf, D.P. (2011). TPF-Interferometer: a decade of development in exoplanet detection 
technology. In SPIE Optical Engineering + Applications, pp. 81510D-81510D. International Society for Optics and Photonics.

Matthews, C.M. (2012). The Arecibo Ionospheric Observatory, Technical report, Congressional Research Service. URL: https://fas.org/sgp/crs/misc/ R40437.pdf

Muhleman, D., Grossman, A. \& Butler, B. (1995). Radar investigations of Mars, Mercury, and Titan. Annual Review of Earth and Planetary Sciences 23, 337-374.

Nan, R. (2008). Introduction to FAST-five hundred meter aperture spherical radio telescope. In Proc. of SPIE, Vol. 7012, pp. 70121E-1.

Nash, D. (2011). The Hyg Database. URL: https:/github.com/astronexus/ HYG-database

Ostro, S. (1993). Planetary radar astronomy. Reviews of Modern Physics 65(4), 1235.

Pettengill, G. \& Dyce, R. (1965). A radar determination of the rotation of the planet Mercury. Nature 206, 1240.

RECONS (2012). The one hundred nearest star systems. The Research Consortium on Nearby Stars. URL: http://www.recons.org/TOP100. posted.htm

Reid, I., Gizis, J. \& Hawley, S. (2007). The Palomar/MSU nearby star spectroscopic survey. IV. The luminosity function in the solar neighborhood and M dwarf kinematics. The Astronomical Journal 124 (5), 2721.

Renzetti, N.A., Thompson, T.W. \& Slade, M.A. (1988). Relative planetary radar sensitivities: Arecibo and Goldstone. Technical Report TDA 42-94, JPL. URL: http://ipnpr.jpl.nasa.gov/progressreport/42-94/94W.PDF

Rogers, A., Ingalls, R. (1969). Venus: mapping the surface reflectivity by radar interferometry. Science (New York, NY) 165(3895), 797.

Rogstad, D.H. (2005). The SUMPLE algorithm for aligning arrays of receiving radio antennas: coherence achieved with less hardware and lower combining loss. Technical Report 42-162. URL: http://ipnpr.jpl.nasa.gov/ progress_report/42-162/title.htm

Ruze, J. (1966). Antenna tolerance theory - a review. Proceedings of the IEEE 54(4), 633-640.

Rzhiga, O. (1985). Radar studies of other planetary systems? Astronomicheskii Zhurnal 62, 500-505. Translation into English by R.B. Rodman at http://adsabs.harvard.edu/abs/1985SvA....29..290R.

Scheffer, L. (2005). A scheme for a high-power, low-cost transmitter for deep space applications. Radio Science 40(5), RS5012.

Shao, M., Catanzarite, J. \& Pan, X. (2010). The synergy of direct imaging and astrometry for orbit determination of exo-earths. Astrophysical Journal 720(1), 357

Simpson, R., Harmon, J., Zisk, S., Thompson, T. \& Muhleman, D. (1992). Radar determination of Mars surface properties. Mars 1, 652-685.

Stacy, N., Campbell, D. \& Ford, P. (1997). Arecibo radar mapping of the lunar poles: a search for ice deposits. Science 276(5318), 1527-1530.

Thompson, T. (1987). High-resolution lunar radar map at 70-cm wavelength. Earth, Moon and Planets 37(1), 59-70.

Tuomi, M., et al. (2012). Signals embedded in the radial velocity noise: periodic variations in the $\tau$ Ceti velocities. Astronomy and Astrophyics 551(3), A79. URL: http://www.aanda.org/articles/aa/abs/2013/03/ aa20509-12/aa20509-12.html.

Williams, F. (1985). A radar for the exploration of exstrasolar planets. Proceedings of the IEEE 73(2), 355-361. Note: The misspelling in the title is in the original.

Yelle, L. (1979). The learning curve: historical review and comprehensive survey. Decision Sciences 10(2), 302-328.

Zisk, S., Pettengill, G. \& Catuna, G. (1974). High-resolution radar maps of the lunar surface at $3.8-\mathrm{cm}$ wavelength. Earth, Moon and Planets 10(1), $17-50$. 